\title{
Vascular catheter colonization: surveillance based on culture of needleless connectors
}

María Jesús Pérez-Granda ${ }^{2,5,6^{*}}$, María Guembe ${ }^{1,3,6^{*}}$, Raquel Cruces ${ }^{1,3}$ and Emilio Bouza ${ }^{1,4,5}$

\begin{abstract}
Background: Superficial culture has a high negative predictive value in the assessment of catheter tip colonization (CC) and catheter-related bloodstream infection (C-RBSI). However, the process of hub culture requires the hubs to be swabbed, and this carries a risk of dislodging the biofilm. At present, most catheter hubs are closed by needleless connectors (NCS) that are periodically replaced. Our objective was to compare the yield of SC (skin + hub culture) with that of skin + NC culture in the assessment of CC and C-RBSI.

Methods: During 5 months, we included the patients on the Major Heart Surgery ICU when a central venous catheter (CVC) remained in place $\geq 7$ days after insertion. SCs were taken simultaneously when the NC was withdrawn and processed by the semi-quantitative method, even when the catheter was not removed. All catheter tips were cultured. All NCs belonging to a single catheter lumen were individually flushed with $100 \mu \mathrm{l}$ of brain-heart infusion (BHI) broth. We considered the lumen to be colonized when $\geq 1 \mathrm{NC}$ culture from the lumen flush was positive. We collected a total of 60 catheters.

Results: The overall CC rate was $15.0 \%$, and we confirmed two episodes of C-RBSI. The validity values after the comparison of SCs with skin + NC culture for prediction of CC were the following: sensitivity $66.7 \%$ vs. $77.8 \%$, and negative predictive value $93.6 \%$ vs. $93.1 \%$. The sensitivity and negative predictive value for prediction of C-RBSI was $100 \%$ for both SC and skin + NC culture.
\end{abstract}

Conclusion: The combination of skin and flushed NC culture can be an alternative to conventional SC for ruling out CC and C-RBSI.

Keywords: Needleless connectors, Flushing, Superficial culture, Hub culture, Catheter colonization, Catheter-related bloodstream infection

\section{Background}

The conventional conservative diagnostic method based on superficial culture requires a surface culture of the skin surrounding the catheter insertion site and a surface culture of the inside of each catheter hub [1]. This procedure has been used in several populations to select patients at risk of catheter-related bloodstream infection (C-RBSI) and to rule out the catheter as the source of the bloodstream infection [2-6]. However, the hub culture process requires needleless connectors ( $\mathrm{NCs}$ ) to be removed so that the swab cab be rubbed along the inside

\footnotetext{
*Correspondence: massus@hotmail.es; mariaguembe@hotmail.com ${ }^{2}$ Cardiac Surgery Postoperative Care Unit, Hospital General Universitario Gregorio Marañón, Madrid, Spain

${ }^{1}$ Department of Clinical Microbiology and Infectious Diseases, Hospital General Universitario Gregorio Marañón, Madrid, Spain

Full list of author information is available at the end of the article
}

of the lumen. The consequent manipulation of the hub can dislodge the biofilm and potentially trigger bloodstream infection [7-9].

A recent study by our group showed that culture (by instillation of brain-heart infusion broth) of the NCs used to close the catheter hubs combined with skin culture, can be an alternative and safer procedure for prediction of catheter colonization and ruling out C-RBSI [10]. However, data on superficial hub culture were not available and, therefore, the yield of conventional superficial culture (skin + hubs) could not be compared with that of skin $+\mathrm{NC}$ cultures for prediction of catheter colonization and C-RBSI. The aim of the present study was to compare the validity values of conventional superficial culture (skin + hubs) with those of skin + NC cultures for catheter tip colonization and C-RBSI. 


\section{Methods}

\section{Setting}

The Major Heart Surgery ICU (MHS-ICU) in our hospital is a 14-bed post-surgical unit for all adult patients who have undergone a major cardiac surgical procedure. Patients admitted to the MHS-ICU during the study period (2 July 2015 to 23 October 2015) were included in the study when a central catheter remained in place $\geq 7$ days after insertion.

\section{Laboratory procedure}

Following the manufacturer's instructions, NCs (CLAVE ${ }^{\text {тм }}$ systems, ICU Medical, Inc., San Clemente, CA, USA) were changed every 7 days and cultured. Simultaneously, superficial culture (from the skin surrounding the catheter insertion site and from the inside of the hubs) was also taken when the NC was withdrawn, even when the catheter was not removed. When the catheter was removed, the last set of NCs and hub cultures was also obtained. Superficial cultures were processed following standard semi-quantitative microbiological techniques [1]. All groups of NCs belonging to a single catheter lumen were individually flushed with $100 \mu \mathrm{l}$ of brain-heart infusion broth (Fig. 1). We considered the lumen colonized when $\geq 1$ culture was positive. The number of cultured NCs varied depending on the number of lumens in each catheter (1-5 lumens).

Catheter tips were withdrawn when clinically indicated, and upon withdrawal they were cultured using the roll-plate (Maki) technique and sonicated onto a blood agar plate [11]. The microorganisms recovered were identified using standard microbiological methods and MALDI-TOF [12].

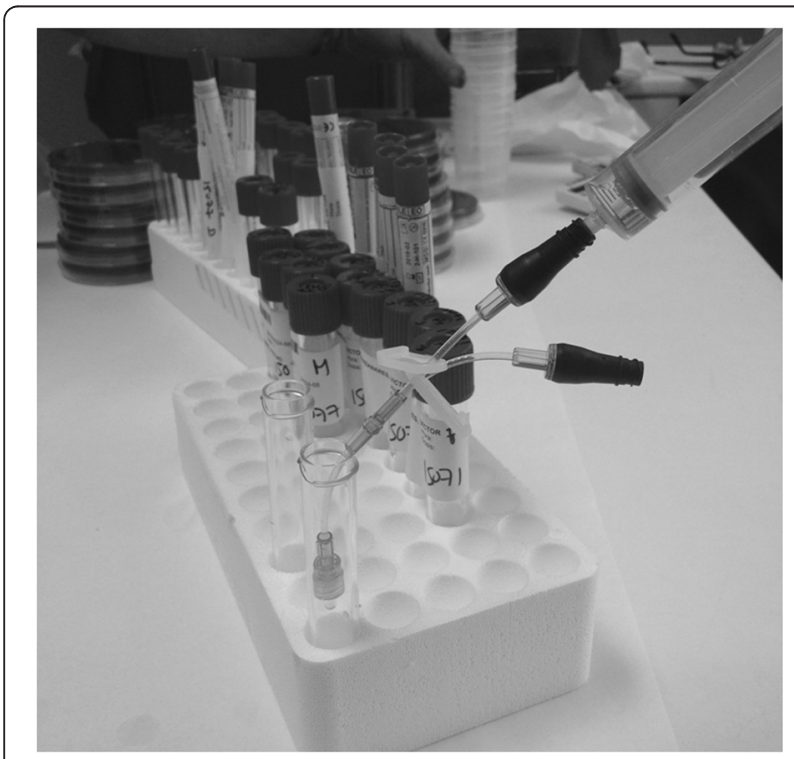

Fig. 1 Laboratory procedure for needleless connector (NC) flushing
We also followed a pre-established protocol to record patient characteristics, underlying diseases, comorbidity factors, severity of illness scores (e.g., acute physiology and chronic health evaluation (APACHE) II), the maximum severity reached until catheter withdrawal, and microbiological data from blood cultures.

\section{Definitions}

\section{Catheter tip colonization}

Isolation of either $\geq 15$ colony forming units (cfu)/plate using Maki's semi-quantitative technique or $\geq 100 \mathrm{cfu} /$ catheter by sonication.

\section{Skin colonization}

Isolation of $\geq 15 \mathrm{cfu} /$ plate by semi-quantitative culture.

\section{Hub colonization}

Isolation of $\geq 15 \mathrm{cfu} /$ plate by semi-quantitative culture.

\section{Closed needleless connector colonization}

Isolation of $\geq 1 \mathrm{cfu} /$ plate in at least one connector in the qualitative culture.

\section{Overall lumen colonization}

When $\geq 1$ NC became positive at any point during surveillance.

\section{C-RBSI}

We considered a C-RBSI episode to be confirmed when the same microorganism was isolated both in peripheral blood cultures and in the catheter tip. The gold standard for confirmation of catheter colonization was positivity of the catheter tip culture either using Maki's semiquantitative technique ( $\geq 15 \mathrm{cfu} /$ plate) or by sonication ( $\geq 100 \mathrm{cfu} /$ segment). To calculate the validity values of superficial culture (skin + hub) and skin $+\mathrm{NC}$ culture for prediction of catheter colonization, we used a positive catheter tip with $\geq 15 \mathrm{cfu} /$ plate of any microorganism as the gold standard.

\section{Statistical analysis}

Values are expressed as the mean (SD) or median (IQR) for continuous variables and as percentages, with the $95 \%$ confidence interval (95\% CI), when applicable, for categorical variables. Categorical variables were evaluated using the chi-square test or the two-tailed Fisher exact test. Statistical significance was set at $p<0.05$ (two-tailed). We calculated the validity values of the superficial culture (skin + hubs) and skin $+\mathrm{NC}$ culture by comparing them with the gold standard of colonization and also with the results obtained by culture of the superficial samples. The sensitivity, specificity, and positive and negative predictive values, with $95 \%$ CI, were calculated using EPIDAT 3.1. Accuracy 
was defined as the sum of true positive and true negative results. The power analysis was calculated for the negative predictive value and sensitivity. Statistical analysis was performed using IBM SPSS Statistics for Windows, Version 21.0 (IBM Corp, Armonk, NY, USA).

\section{Ethics}

The study was approved by the local ethics committee of Hospital General Universitario Gregorio Marañón and the ethics committee waived the need for informed consent.

\section{Results}

We collected 60 catheters from 34 patients who had a catheter inserted for $\geq 7$ days. The mean (SD) age was 63.7 (11.9) years. The main underlying conditions were congestive heart failure $(67.6 \%)$ and diabetes mellitus (35.3\%). The mean (SD) comorbidity index, APACHE II at inclusion, and EuroScore were 2.6 (1.7), 8.3 (3.3), and 6.7 (2.5), respectively. The main reason for catheter withdrawal was end of use (63.3\%), followed by suspicion of infection $(25.0 \%)$, and other reasons (11.7\%). We confirmed 2 episodes of C-RBSI (2.2 episodes/1,000 catheter days). Other patient and catheter data are detailed in Table 1 . The crude mortality rate of the study population was $26.7 \%$.

The overall catheter tip colonization rate was $15.0 \%$ (9/60). Out of the 60 catheters, $29(48.3 \%)$ skin and/or $\mathrm{NC}$ and/or hub cultures were never positive. In the remaining 31 (51.7\%), skin and/or NCs and/or hubs were positive at least once.

Table 2 shows the validity values for skin $+\mathrm{NC}$ cultures and superficial culture for prediction of catheter colonization and C-RBSI. Skin + NC cultures had $77.8 \%$ sensitivity and a $93.1 \%$ negative predictive value for catheter colonization compared with $66.7 \%$ and $93.6 \%$ for superficial culture $(p=0.02)$. The accuracy for skin $+\mathrm{NC}$ cultures and superficial culture was 7 true positive/27 true negative, and 6 true positive/44 true negative, respectively. NC cultures had the same negative predictive value for C-RBSI (100\%). Table 3 shows the microorganisms isolated from the colonized central venous catheters.

\section{Discussion}

The new procedure we describe for ruling out catheter colonization and C-RBSI based on the combination of skin and NC cultures showed, at least, no inferiority to the conventional superficial culture. Guidelines recommend using superficial culture (skin and hub) as a useful conservative procedure for the diagnosis of catheter tip colonization and C-RBSI [1]. The recommendation is based on the pathogenesis of catheter colonization, which occurs by progression of microorganisms to the tip of the catheter along either the inner surface ( $\geq 7$ days
Table 1 Main patient and catheter characteristics

\begin{tabular}{|c|c|}
\hline Characteristic & $\mathrm{N}(\%)$ \\
\hline \multicolumn{2}{|l|}{ Patients ( $\mathrm{N}=34)$} \\
\hline Mean (SD) age, years & $63.7(11.9)$ \\
\hline Sex, male/female & $21 / 13$ \\
\hline \multicolumn{2}{|l|}{ Underlying conditions (\%) } \\
\hline Myocardial infarction & $3(8.8)$ \\
\hline Congestive heart failure & $23(67.6)$ \\
\hline ACVA & $6(17.6)$ \\
\hline Chronic obstructive pulmonary disease & $7(11.7)$ \\
\hline Diabetes mellitus & $12(35.3)$ \\
\hline Peptic ulcer disease & $6(17.6)$ \\
\hline Peripheral vascular disease & $3(8.8)$ \\
\hline Renal dysfunction & $7(20.6)$ \\
\hline EuroScore ${ }^{a}$, mean (SD) & $6.7(2.5)$ \\
\hline Charlson comorbidity index, mean (SD) & $2.6(1.7)$ \\
\hline Non-fatal underlying disease, McCabe (\%) & $25(73.5)$ \\
\hline APACHE II at inclusion, mean (SD) & $8.3(3.3)$ \\
\hline Length of ICU stay (days), median (IQR) & 10.5. $(7.7-38.0)$ \\
\hline Crude mortality (\%) & $8(26.7)$ \\
\hline \multicolumn{2}{|l|}{ Catheters $(\mathrm{N}=60)$} \\
\hline \multicolumn{2}{|l|}{ Type } \\
\hline Non-tunneled central venous catheter & $47(78.3)$ \\
\hline Guidewire & $13(21.7)$ \\
\hline \multicolumn{2}{|l|}{ Location } \\
\hline Jugular & $56(93.3)$ \\
\hline Subclavian & $4(6.7)$ \\
\hline Total parenteral nutrition/propofol & $17(28.3)$ \\
\hline \multicolumn{2}{|l|}{ Reasons for catheter withdrawal } \\
\hline End of use & $38(63.3)$ \\
\hline Suspicion of infection & $15(25.0)$ \\
\hline Others & $7(11.7)$ \\
\hline Catheter-days, median (IQR) & $11.0(8.0-20.0)$ \\
\hline Total catheter-days & 906 \\
\hline Catheter colonization (\%) & $9(15.0)$ \\
\hline Catheter colonization/1,000 catheter-days & $9.9 / 1,000$ \\
\hline C-RBSI episodes (\%) & $2(3.3)$ \\
\hline C-RBSI/1,000 catheter-days & $2.2 / 1,000$ \\
\hline
\end{tabular}

$\mathrm{SD}$, standard deviation; IQR, interquartile range; ICU, intensive care unit; $\mathrm{ACVA}$, acute cerebrovascular accident; C-RBSI, catheter-related bloodstream infection ${ }^{a}$ EuroScore, European System for Cardiac Operative Risk Evaluation

of indwelling time) or the outer surface ( $<7$ days of indwelling time) of the catheter. Superficial culture has been used in several populations (e.g., MHS-ICU, oncology, and hemodialysis patients) and helps prevent unnecessary catheter withdrawal [2-6]. However, conventional superficial culture requires swabs to be rubbed 
Table 2 Validity values of skin and NC culture and superficial culture (skin + hubs) for prediction of catheter colonization and C-RBSI

\begin{tabular}{|c|c|c|c|c|c|c|c|c|}
\hline CULTURE & $\begin{array}{c}5 \% \\
95 \% \mathrm{Cl}\end{array}$ & $\begin{array}{c}\mathrm{SP} \% \\
95 \% \mathrm{Cl}\end{array}$ & $\begin{array}{l}\text { PPV\% } \\
95 \% \mathrm{Cl}\end{array}$ & $\begin{array}{l}\text { NPV\% } \\
95 \% \mathrm{Cl}\end{array}$ & $\begin{array}{c}\text { Validity index } \\
95 \% \mathrm{Cl}\end{array}$ & $\begin{array}{c}\text { Prevalence } \\
95 \% \mathrm{Cl}\end{array}$ & $\begin{array}{c}\mathrm{LR}+ \\
95 \% \mathrm{Cl}\end{array}$ & $\begin{array}{c}\text { LR- } \\
95 \% \mathrm{Cl}\end{array}$ \\
\hline \multicolumn{9}{|c|}{ Catheter colonization } \\
\hline Skin+NCs & $\begin{array}{c}77.8 \\
(45.0-100)\end{array}$ & $\begin{array}{c}52.9 \\
(38.3-67.6)\end{array}$ & $\begin{array}{c}22.6 \\
(6.2-38.9)-\end{array}$ & $\begin{array}{c}93.1 \\
(82.2-100)\end{array}$ & $\begin{array}{c}56.7 \\
(43.3-70.0)\end{array}$ & $\begin{array}{c}15.0 \\
(5.1-24.9)\end{array}$ & $\begin{array}{c}1.65 \\
(1.05-2.60)\end{array}$ & $\begin{array}{c}0.42 \\
(0.12-1.46)\end{array}$ \\
\hline Skin+hubs & $\begin{array}{c}66.7 \\
(30.3-100)\end{array}$ & $\begin{array}{c}86.3 \\
(75.8-92.0)\end{array}$ & $\begin{array}{c}46.1 \\
(15.2-77.1)\end{array}$ & $\begin{array}{c}93.6 \\
(85.6-100)\end{array}$ & $\begin{array}{c}83.3 \\
(73.0-93.6)\end{array}$ & $\begin{array}{c}15.0 \\
(5.1-24.9)\end{array}$ & $\begin{array}{c}4.86 \\
(2.12-11.13)\end{array}$ & $\begin{array}{c}0.39 \\
(0.15-0.98)\end{array}$ \\
\hline \multicolumn{9}{|l|}{ C-RBSI } \\
\hline Skin+NCs & $\begin{array}{c}100 \\
(75.0-100)\end{array}$ & $\begin{array}{c}50.0 \\
(36.3-63.7)\end{array}$ & $\begin{array}{c}6.4 \\
(0.0-16.7)\end{array}$ & $\begin{array}{c}100 \\
(98.3-100)\end{array}$ & $\begin{array}{c}51.7 \\
(38.2-65.1)\end{array}$ & $\begin{array}{c}3.3 \\
(0.0-8.7)\end{array}$ & $\begin{array}{c}2.00 \\
(1.55-2.59)\end{array}$ & NA \\
\hline Skin+hubs & $\begin{array}{c}100 \\
(75.00-100)\end{array}$ & $\begin{array}{c}81.0 \\
(70.0-91.9)\end{array}$ & $\begin{array}{c}15.4 \\
(0.0-38.8)\end{array}$ & $\begin{array}{c}100 \\
(98.9-100)\end{array}$ & $\begin{array}{c}76.8 \\
(67.0-86.6)\end{array}$ & $\begin{array}{c}3.3 \\
(0.0-8.7)\end{array}$ & $\begin{array}{c}5.27 \\
(3.10-8.98)\end{array}$ & NA \\
\hline
\end{tabular}

S, sensitivity; SP, specificity; PPV, positive predictive value; NPV, negative predictive value; LR+, positive likelihood ratio; $\mathrm{LR}$-, negative likelihood ratio; $\mathrm{Cl}$, confidence interval; NA, not applicable; C-RBSI, catheter-related bloodstream infection

along the inside of the hubs, thus increasing the risk of dislodging the biofilm and triggering bloodstream infection [7-9].

Our group recently tested an alternative superficial culture procedure for hubs [10]. We hypothesized that as NCs are substituted periodically to decrease catheter colonization, they can be used as an alternative to hub culture for diagnosis, thus avoiding excessive manipulation. The laboratory procedure was performed by flushing $100 \mu \mathrm{l}$ of brain-heart infusion broth through the inside of the NCs before culture. The results showed that NCs combined with skin superficial culture had good sensitivity and negative predictive values for catheter colonization and C-RBSI. However, data were not

Table 3 Microorganisms isolated in colonized catheters

\begin{tabular}{ccc}
\hline Catheter tip & Skin + NCs & Skin + hubs \\
\hline Staphylococcus & Staphylococcus & Staphylococcus \\
epidermidis & epidermidis & epidermidis \\
Staphylococcus & Staphylococcus & Staphylococcus \\
epidermidis & epidermidis & epidis \\
Staphylococcus & Staphylococcus & Staphylococcus \\
epidermidis & epidermidis & epidermidis \\
Cons & - & - \\
Staphylococcus & Staphylococcus & Staphylococcus \\
epidermidis & epidermidis & epidermidis \\
& Moraxella osloensis & Klebsiella pneumoniae \\
Candida albicans & Slebsiella pneumoniae & \\
Staphylococcus & Staphylococcus hominis & - \\
epidermidis & epidermidis & Staphylococcus \\
& epidermidis \\
& Staphylococcus aureus & \\
Staphylococcus & Staphlococcus & \\
epidermidis & Staphylococcus & Staphylococcus \\
Staphylococcus & epidermidis & epidermidis \\
hominis & - & - \\
\hline
\end{tabular}

NCs, needleless connectors; CoNS, coagulase-negative staphylococci compared with those for superficial hub culture, as this approach was not used.

In the present study, we resolved this limitation and found that the sensitivity for predicting catheter colonization using skin $+\mathrm{NC}$ culture compared with conventional superficial culture was $77.8 \%$ vs. $66.7 \%$ $(p=0.02)$. However, both combinations of procedures had better accuracy for the coagulase-negative Staphylococci than for the other etiological origins. Besides, we did not find statistically significant differences between the procedures in the prediction of C-RBSI. The most important finding was that skin $+\mathrm{NC}$ culture had a high negative predictive value for catheter colonization and C-RBSI (93.1 \% and $100 \%$, respectively).

The main limitation of the study was the small sample size and the low rate of catheter tip colonization. However, we calculated the power analysis for the negative predictive value and sensitivity of the obtained results as follows: negative predictive value $4.0 \%$ (93.1-93.6 \%); sensitivity $28.0 \%$ (77.8-66.7 \%). The obtained power for sensitivity was enough to detect statistical significant differences $(p=0.02)$. We consider that our results are promising for the substitution of superficial hub culture by culture of $\mathrm{NC}$ instillations. Future clinical studies are needed to assess the impact of this procedure in the prevention of catheter tip colonization.

\section{Conclusions}

We demonstrated that superficial skin culture combined with $\mathrm{NC}$ culture was not inferior to conventional superficial culture of skin and hubs in the selection of patients with no risk of catheter colonization or C-RBSI. Moreover, the new diagnostic approach is easier and less invasive to apply.

\section{Acknowledgments}

We thank Thomas O'Boyle for his help in the preparation of the manuscript. M. Guembe is supported by the Miguel Servet Program (ISCIII-MICINN, CP13/ 00268) from the Health Research Fund (FIS), of the Carlos III Health Institute (ISCIII), Madrid Spain, partially financed by the by the European Regional 
Development Fund (FEDER) "A way of making Europe". The funders had no role in study design, data collection and analysis, decision to publish, or preparation of the manuscript.

\section{Authors' contributions}

MJPG participated in the study conception and design, carried out the analysis and interpretation of the data, and carried out the manuscript writing. MG participated in the study conception and design, performed the statistical analysis, and was involved in drafting the manuscript. RC carried out the sample collection and data acquisition, and was involved in drafting the manuscript. EB participated in the study conception and revised the manuscript critically for important intellectual content. All authors read and approved the final manuscript

\section{Competing interests}

The authors declare that they have no competing interests.

\section{Author details}

${ }^{1}$ Department of Clinical Microbiology and Infectious Diseases, Hospital General Universitario Gregorio Marañón, Madrid, Spain. ${ }^{2}$ Cardiac Surgery Postoperative Care Unit, Hospital General Universitario Gregorio Marañón, Madrid, Spain. ${ }^{3}$ Instituto de Investigación Sanitaria Gregorio Marañón (liSGM), Madrid, Spain. ${ }^{4}$ Medicine Department, School of Medicine, Universidad Complutense de Madrid, Madrid, Spain. ${ }^{5} \mathrm{CIBER}$ de Enfermedades Respiratorias-CIBERES (CB06/06/0058), Madrid, Spain. ${ }^{6}$ Hospital General Universitario "Gregorio Marañón", C/Dr. Esquerdo, 46, 28007 Madrid, Spain.

Received: 29 February 2016 Accepted: 4 May 2016

\section{Published online: 28 May 2016}

\section{References}

1. Mermel LA, Allon M, Bouza E, Craven DE, Flynn P, O'Grady NP, et al. Clinical practice guidelines for the diagnosis and management of intravascular catheter-related infection: 2009 Update by the Infectious Diseases Society of America. Clin Infect Dis. 2009;49(1):1-45.

2. Bouza E, Alvarado N, Alcala L, Perez MJ, Rincon C, Munoz P. A randomized and prospective study of 3 procedures for the diagnosis of catheterrelated bloodstream infection without catheter withdrawal. Clin Infect Dis. 2007:44(6):820-6.

3. Bouza E, Munoz P, Burillo A, Lopez-Rodriguez J, Fernandez-Perez C, Perez MJ, et al. The challenge of anticipating catheter tip colonization in major heart surgery patients in the intensive care unit: are surface cultures useful? Crit Care Med. 2005;33(9):1953-60.

4. Bouza E, Rojas L, Guembe M, Marin M, Anaya F, Luno J, et al. Predictive value of superficial cultures to anticipate tunneled hemodialysis catheterrelated bloodstream infection. Diagn Microbiol Infect Dis. 2014;78(3):316-9.

5. Cercenado E, Ena J, Rodriguez-Creixems M, Romero I, Bouza E. A conservative procedure for the diagnosis of catheter-related infections. Arch Intern Med. 1990;150(7):1417-20.

6. Guembe M, Martin-Rabadan P, Echenagusia A, Camunez F, RodriguezRosales $G$, Simo G, et al. Value of superficial cultures for prediction of catheter-related bloodstream infection in long-term catheters: a prospective study. J Clin Microbiol. 2013;51(9):3025-30.

7. Bouza E, Munoz P, Lopez-Rodriguez J, Jesus Perez M, Rincon C, Martin Rabadan P, et al. A needleless closed system device (CLAVE) protects from intravascular catheter tip and hub colonization: a prospective randomized study. J Hosp Infect. 2003;54(4):279-87.

8. Raad II. The pathogenesis and prevention of central venous catheter-related infections. Middle East J Anesthesiol. 1994:12(4):381-403.

9. Sitges-Serra A, Pi-Suner T, Garces JM, Segura M. Pathogenesis and prevention of catheter-related septicemia. Am J Infect Control. 1995;23(5):310-6.

10. Pérez-Granda MJ GM, Cruces R, Barrio JM, Bouza E. Assessment of central venous catheters colonization using surveillance culture of withdrawn connectors and insertion site skin. Crit Care. 2016. Published online 02 February 2016.

11. Maki DG, Weise CE, Sarafin HW. A semiquantitative culture method for identifying intravenous-catheter-related infection. N Engl J Med. 1977;296(23):1305-9.

12. Mohr-O'Hara CWM MMJ. Manual and automated systems for detection and identification of microorganisms. In: Murray PR, Jorgesen JH, Pfaller MA, Yolken RH (eds) Manual of Clinical Microbiology. Washington, DC: American Society for Microbiology; 2003. p. 185-207.

\section{Submit your next manuscript to BioMed Central and we will help you at every step:}

- We accept pre-submission inquiries

- Our selector tool helps you to find the most relevant journal

- We provide round the clock customer support

- Convenient online submission

- Thorough peer review

- Inclusion in PubMed and all major indexing services

- Maximum visibility for your research

Submit your manuscript at www.biomedcentral.com/submit
) Biomed Central 